\title{
Association between clot composition and stroke origin in mechanical thrombectomy patients: analysis of the Stroke Thromboembolism Registry of Imaging and Pathology
}

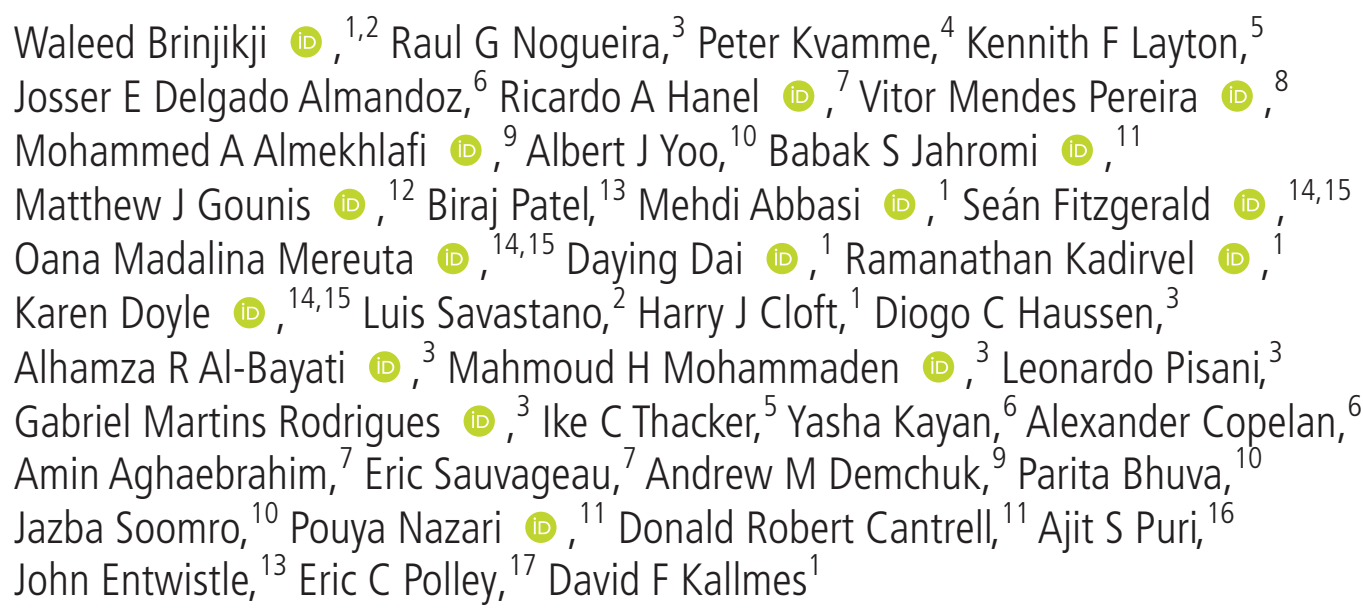

\section{ABSTRACT}

Background We retrospectively evaluated the composition of retrieved clots from ischemic stroke patients to study the association between histological composition and stroke etiology

Methods Consecutive patients enrolled in the Stroke Thromboembolism Registry of Imaging and Pathology (STRIP) were included in this study. All patients underwent mechanical thrombectomy and retrieved clots were sent to a central core lab for processing. Histological analysis was performed using martius scarlet blue (MSB) staining, and quantification for red blood cells (RBCs), white blood cells (WBCs), fibrin and platelets was performed using Orbit Image Software. A Wilcoxon test was used for continuous variables and $\chi^{2}$ test for categorical variables.

Results 1350 patients were included in this study. The overall rate of Thrombolysis In Cerebral Infarction ( $\mathrm{TICI}$ ) 2c/3 was $68 \% .501$ patients received tissue plasminogen activator (tPA) (37\%). 267 patients (20\%) had a large artery atherosclerosis (LAA) source, 662 (49\%) a cardioembolic (CE) source, 301 (22\%) were cryptogenic, and the remainder had other identifiable sources including hypercoagulable state or dissection. LAA thrombi had a higher mean RBC density $(46 \pm 23 \%$ vs $42 \pm 22 \%, p=0.01)$ and a lower platelet density ( $24 \pm 18 \%$ vs $27 \pm 18 \%, p=0.03$ ) than CE thrombi. Clots from dissection patients had the highest mean RBC density $(50 \pm 24 \%)$ while clots from patients with a hypercoagulable state had the lowest mean RBC density $(26 \pm 21 \%)$.

Conclusions Our study found statistically significant but clinically insignificant differences between clots of CE and LAA etiologies. Future studies should emphasize molecular, proteomic and immunohistochemical characteristics to determine links between clot composition and etiology.

\section{INTRODUCTION}

Given the increased utilization of mechanical thrombectomy for the treatment of acute ischemic stroke secondary to large vessel occlusion, there has been growing interest in the analysis of retrieved thrombi. ${ }^{1-7} \mathrm{~A}$ number of studies have suggested that histopathological analysis can provide some valuable insights into stroke etiology. ${ }^{8-11}$ Many of these studies have relied on traditional hematoxylin and eosin (H\&E) and martius scarlet blue (MSB) stains to quantify the proportion of clot components including red blood cells (RBCs), fibrin, white blood cells (WBCs) and platelets. Other studies have sought to examine the role that immunohistochemistry and more advanced proteomic analyses can play in differentiating between various clot etiologies.

Understanding that there is a reliable histological signature of retrieved thrombi is important because we can then potentially help to discern between emboli which are more likely to be cardiac in etiology versus those of a large artery atherosclerosis (LAA) etiology, and perhaps influence secondary prevention strategies accordingly. If a large study were to find no such link, then it would suggest that the field should focus on more advanced analyses of clot composition including proteomic signatures, molecular analyses and immunohistochemistry. In order to study the association between stroke etiology and clot histological composition, we studied patients included in the 
Stroke Thromboembolism Registry of Imaging and Pathology (STRIP). We hypothesized that clots retrieved from patients who had an LAA source would have a higher proportion of platelets and WBCs than those from a cardiac source.

\section{PATIENTS AND METHODS Patient population}

Consecutive patients enrolled in the STRIP Registry from September 2016 to December 2019 were included. The study was institutional review board approved and waiver of consent was granted. Patients were included if they were $>18$ years of age, had undergone mechanical thrombectomy treatment for acute ischemic stroke, and clot material was retrieved.

\section{Clot processing and histological characterization}

Each embolus was immediately fixed in 10\% phosphate buffered formalin. Emboli were shipped to a central core laboratory for standard tissue processing and embedded in paraffin. The formalin-fixed paraffin-embedded clot material was cut into 3-5 $\mu \mathrm{m}$ sections. Representative slides from each clot were stained with H\&E and MSB. Representative MSB-stained slides were sent for whole slide scanning (Aperio ScansScope AT-Turbo, Leica Biosystems). Histological quantification was performed using Orbit Image Analysis Software (www.Orbit.bio) as per the standard operating procedure. ${ }^{12}$ Details of the methodology for Orbit image analysis has been previously described.

\section{Data collection}

Data regarding patient demographics, clinical presentation, treatment strategies, outcome, imaging findings, and stroke pathogenesis were collected using a data abstraction form. This is provided in the supplement. For the purpose of this study, we collected data on demographics, the use of tissue plasminogen activator (tPA), location of the occluded vessel, number of passes, and final Thrombolysis in Cerebral Infarction (TICI) score. Stroke work-up was performed depending on each institution's protocol. In general, this consisted of a form of carotid vascular imaging for detection of stenosis (ie, CT angiography of the neck or ultrasound of the neck) as well as echocardiography (transesophageal or transthoracic) and cardiac monitoring. All stroke etiology work-ups were performed by vascular neurologists at the treating institution.

\section{Statistical analysis}

The mean (SD) of RBCs, fibrin, platelets and WBCs for each stroke etiology subgroup were calculated. In order to model clot composition as a categorical variable, we also performed analyses based on the dominant component in a clot. If the density of a component was $50 \%$ or higher, then the clot was considered 'rich' in that component (ie, RBC-rich, platelet-rich, fibrin-rich, etc).

Categorical variables were compared using the $\chi^{2}$ test. Continuous variables were compared using an analysis of variance (ANOVA) test across all groups as well as Student's t-test for each pair. We also performed a receiver operator characteristic (ROC) analysis to determine if any threshold of RBC, WBC, fibrin and platelet density could be used to differentiate cardioembolic (CE) versus LAA clots. All statistical correlations were assessed using JMP 14.0 (www.jmp.com, Cary, NC).

A machine learning classification algorithms were estimated using the histological characterizations as the features in the algorithm. The classification algorithm was to differentiate $\mathrm{CE}$ versus LAA subgroups. The stacked ensemble framework ${ }^{13}$ was used in the H2o.ai R package. ${ }^{14}$ The algorithm in the ensemble included regularized logistic regression, gradient boosted machines, deep neural networks, and extremely randomized forests. Fivefold cross-validation estimates of the area under the ROC curve and the area under the precision-recall curve were estimated to evaluate classification performance.

\section{RESULTS}

\section{Patient population}

A total of 1350 patients were included, with a mean age of $68.5 \pm 13.5$ years. The overall rate of TICI $2 \mathrm{c} / 3$ was $68 \%$ (919/1350). A total of 501 patients received tPA (37\%) and there was no difference in the proportion of RBCs, fibrin or platelets between clots which were and were not treated with tPA $(\mathrm{p}=0.89, \mathrm{p}=0.98$ and $\mathrm{p}=0.75$, respectively). Regarding stroke etiology, 267 patients (20\%) had an LAA source, $662(49 \%)$ a CE source, 301 (23\%) were cryptogenic, 26 patients (2\%) had dissection, and 23 patients (2\%) had a known hypercoagulable state. The mean number of passes was $2 \pm 2$.

\section{Histology results}

The histological results are summarized in table 1 . There were significant differences in RBC density across groups ranging from

Table 1 Clot composition between groups

\begin{tabular}{lccccccc}
\hline & Cardioembolic & Large artery atherosclerosis & Other & ESUS & Dissection & Hypercoagualable state & $\begin{array}{l}\text { P value across } \\
\text { groups }\end{array}$ \\
\hline Number of cases & 662 & 267 & 71 & 301 & 26 & 23 & \\
\hline Percentage of clot components & & & & & & & \\
\hline Mean (SD) RBC & $42(22)$ & $46(23)$ & $45(23)$ & $41(24)$ & $50(24)$ & $26(21)$ & 0.0004 \\
\hline Mean (SD) WBC & $4(2)$ & $4(2)$ & $3(2)$ & $4(2)$ & $4(3)$ & $3(3)$ & 0.223 \\
\hline Mean (SD) fibrin & $27(16)$ & $26(15)$ & $25(15)$ & $25(14)$ & $27(16)$ & $36(17)$ & 0.01 \\
\hline Mean (SD) platelets & $27(18)$ & $24(18)$ & $26(22)$ & $29(21)$ & $18(13)$ & $35(27)$ & 0.002 \\
\hline Dominant clot component & & & & & & & \\
\hline RBC N (\%) & $252(38)$ & $117(44)$ & $30(42)$ & $117(39)$ & $11(44)$ & $3(13)$ & \\
\hline Fibrin N (\%) & $73(11)$ & $22(8)$ & $5(7)$ & $22(7)$ & $3(12)$ & $4(17)$ & 0.08 \\
\hline Platelets N (\%) & $79(12)$ & $26(10)$ & 9 & $49(16)$ & $0(0)$ & $6(26)$ & 0.28 \\
\hline
\end{tabular}

ESUS, embolic stroke of undetermined source; RBC, red blood cells; WBC, white blood cells. 


\section{Red Blood Cells}



Fibrin

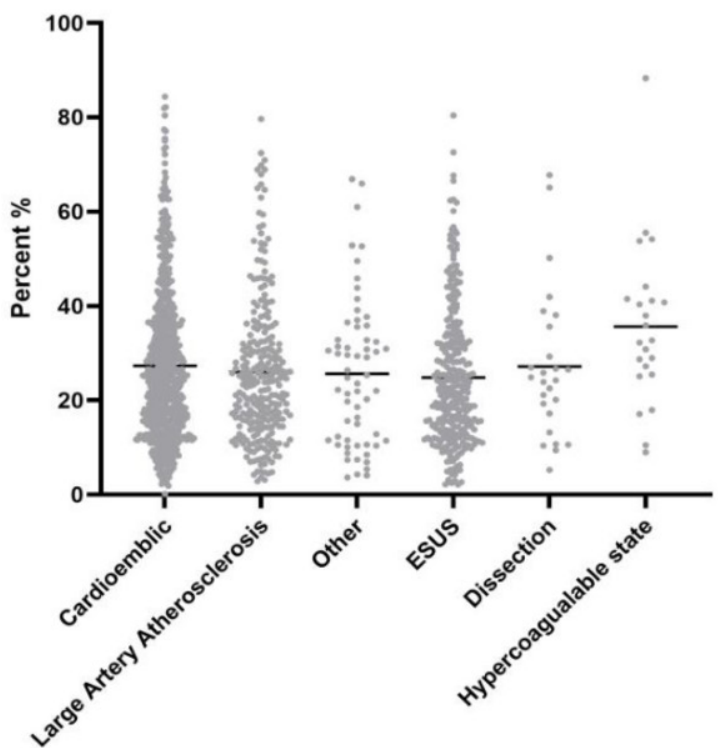

White Blood Cells

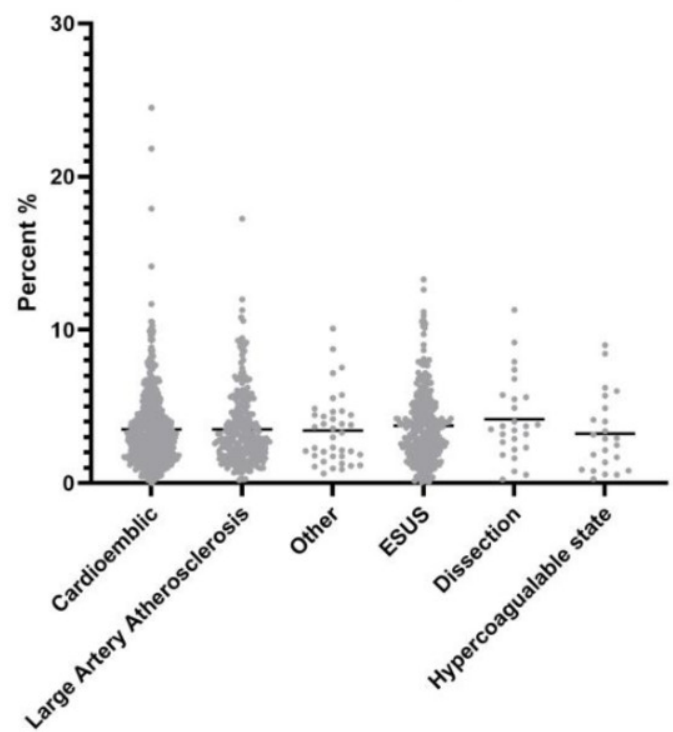

Platelets



Figure 1 Scatter plot showing each component of clot for various stroke etiologies.

$26 \pm 21 \%$ for patients with hypercoagulable state to $50 \pm 24 \%$ for patients with dissection $(\mathrm{p}=0.0004)$. Patients with LAA clots had a higher mean RBC density than those with CE clots $(46 \pm 23 \%$ vs $42 \pm 22 \%, p=0.01)$. LAA clots also had a significantly higher RBC density than embolic stroke of undetermined source (ESUS) clots $(46 \pm 23 \%$ vs $41 \pm 24 \%, \mathrm{p}=0.02)$. All groups had a significantly higher RBC composition than clots from patients with hypercoagulable states $(p<0.001)$. All other differences in RBC density were insignificant across groups. Figure 1 depicts the distribution of each component across different stroke etiologies.

A total of 530 clots were considered RBC-rich (39\%). Hypercoagulable state clots were the least likely to be RBC-rich (13\%) while LAA and dissection clots were the most likely to be RBCrich (44\%). There was no difference in the proportion of patients with RBC-rich clots between the LAA and CE groups (44\% vs
$38 \%, \mathrm{p}=0.11)$. Hypercoagulable state clots were less likely to be RBC-rich than all other groups $(\mathrm{p}<0.01)$.

Mean WBC density was similar across all groups ranging from $3 \pm 3 \%$ for hypercoagulable state clots to $4 \pm 2 \%$ for other groups $(p=0.223)$. There were no significant differences in any of the pairwise comparisons.

There were significant differences in mean fibrin density across groups ranging from $25 \pm 14 \%$ for cryptogenic stroke patients to $36 \pm 17 \%$ from those with hypercoagulable states $(p=0.01)$. Patients with CE clots had a slightly higher mean fibrin density than those with ESUS $(p=0.02)$. Patients with a hypercoagulable state had a significantly higher fibrin density than all other groups $(\mathrm{p}<0.05$ for each pair-wise comparison). All other differences in fibrin density were insignificant across groups. 
A total of 129 clots were considered fibrin-rich (9.6\%). Hypercoagulable state clots were the most likely to be fibrin-rich (17\%) while ESUS clots were the least likely (7\%). There was no difference in the proportion of patients with fibrin-rich clots between the LAA and CE groups ( $8 \%$ vs $11 \%, p=0.23$ ). There was a significant difference in the proportion of patients with fibrin-rich clots across groups $(\mathrm{p}=0.28)$.

There were significant differences in mean platelet density across groups ranging from $18 \pm 13 \%$ for dissection patients to $35 \pm 27 \%$ for hypercoagulable state patients $(p=0.002)$. CE patients had a higher platelet density than LAA patients $(27 \pm 18 \%$ vs $24 \pm 18 \%, \mathrm{p}=0.03)$ and a higher platelet density than dissection patients $(27 \pm 18 \%$ vs $18 \pm 13 \%)$. ESUS patients had a higher platelet density than LAA patients $(29 \pm 21 \%$ vs $24 \pm 18 \%, \mathrm{p}=0.005)$. Hypercoagulable state patients had a higher platelet density than all other groups $(\mathrm{p}<0.05$ for all comparisons).

A total of 169 clots were platelet-rich (13\%). The proportion of platelet-rich clots ranged from $10 \%$ for LAA clots to $26 \%$ for hypercoagulable state clots $(\mathrm{p}=0.02)$. There was no difference in the proportion of platelet-rich clots between CE and LAA patients $(12 \%$ vs $10 \%, \mathrm{p}=0.36)$.

On ROC analysis, the area under the curve (AUC) for RBC density in differentiating CE from LAA clots was 0.55 ; the AUC for WBC density was 0.50 ; the AUC for fibrin density was 0.52 ; and the AUC for platelet density was 0.55 .

\section{Machine learning classification algorithm results}

The stacked ensemble for the classification algorithm differentiating CE from LAA had a fivefold cross-validated AUC of 0.55 (area under the precision-recall curve of 0.33).

\section{Other clinical results}

On ANOVA analysis, there was no difference in the mean number of passes based on stroke etiology. The mean number of passes was $1.9 \pm 1.4$ for CE, $2.0 \pm 1.5$ for LAA, $2.0 \pm 1.7$ for ESUS source, $2.7 \pm 2.3$ for hypercoagulable source, and $2.1 \pm 1.4$ for dissection $(p=0.19)$. First pass TICI $2 c / 3$ for these groups were, respectively, $42.2 \%, 37.9 \%, 39.3 \%, 26.9 \%$, and $43.5 \%$ $(\mathrm{p}=0.18)$. Overall TICI $2 \mathrm{c} / 3$ rates were $70.5 \%, 65.9 \%, 66.5 \%$, $61.5 \%$, and $69.6 \%$, respectively $(\mathrm{p}=0.07)$. Embolization to previously non-affected territories was significantly higher for dissection patients $(13.0 \%)$ when compared with CE (2.1\%), LAA (3.0\%), ESUS (2.7\%), and hypercoagulable state (4\%) patients $(\mathrm{p}=0.02)$.

\section{DISCUSSION}

Our study examining clot composition in a large cohort of ischemic stroke patients and its association with stroke etiology demonstrated a number of interesting findings. First, LAA clots had a higher mean RBC density and a lower mean platelet density than CE clots. On ROC analysis, it seems that identification of a reliable threshold with a high AUC for differentiating clots from these two etiologies based on composition analysis alone is not possible. The lack of a reliable histological biomarker on MSB between these two stroke etiologies suggests that conventional histological analyses looking at cellular composition do not provide insights into stroke etiology in cryptogenic cases. One interesting association that we found in our study was that thrombi from patients with a known hypercoagulable state had statistically and clinically significant differences in the mean RBC density and higher fibrin and platelet density than clots from any other known source. Ultimately, we feel these findings are important because they suggest that future investigations into what clues clots can provide for stroke etiology should be focused on more advanced testing such as proteomic composition, structural analyses and immunohistochemical markers rather than conventional histology.

A number of previous studies have examined the histological characteristics of LAA and CE clots to see if they could provide any insight into the source of cryptogenic strokes. Prior studies examining correlations between thrombus composition and stroke pathogenesis have focused specifically on RBCs, WBCs and fibrin/platelet compositions with inconclusive results. A study of 187 patients by Sporns et al found that CE emboli had few RBCs and more fibrin/platelets than non-CE emboli; however, they did not account for fibrin and platelet compositions. ${ }^{11}$ Meanwhile, a study by Kim et al of 37 patients found that $\mathrm{CE}$ clots were actually more likely to have a high $\mathrm{RBC}$ composition compared with those related to LAA. ${ }^{15}$ A similar study by Boeckh-Behrens et al of 145 patients found that CE emboli had higher proportions of fibrin and platelets and fewer RBCs than non-CE emboli. ${ }^{9}$ Lastly, a prior study from our group found that LAA clots were more likely to be platelet-rich and had a slightly lower RBC density than CE strokes. ${ }^{10}$ Our study of over 1300 patients showed no findings which allowed us to reliably distinguish CE and LAA clots.

Overall, the findings from our study and the disparate results from multiple prior studies suggest that routine histological staining with H\&E or MSB probably will not allow us to differentiate clots of different etiologies and will likely play no role in determining stroke etiology in ESUS. However, some groups have looked into more sophisticated analyses of clot composition to help identify and differentiate stroke etiologies. Recently, Munoz et al have demonstrated the feasibility of mass spectrometry-based proteomic profiling of thrombotic material obtained by embolectomy in ischemic stroke and have help characterize the clot proteome. ${ }^{16}$ Meanwhile, other groups are examining the role of immunohistochemistry markers staining for proteins such as von Willebrand factor (vWF) and ADAMTS13 to point towards stroke etiology. ${ }^{17}$ Based on the findings from our study, we feel that histological analyses to determine stroke etiology are likely insufficient and future research should be directed toward more sophisticated analyses focused on immunohistochemistry, proteomics and molecular analyses.

One other feature that we did not specifically examine is the association between clot organization and structure and stroke etiology. While the relative proportion of each cellular component may not provide clues into stroke etiology, the way in which the components are organized might help in differentiation of the embolic source. For example, in a study by Di Meglio et al using scanning electron microscopy, the authors found that ischemic stroke thrombi had an outer shell of densely compacted fibrin, $\mathrm{vWF}$ and platelets that made them refractory to thrombolysis. ${ }^{18}$ Perhaps we may find that clots from CE sources and those from LAA sources are organized differently from one another with different surface components.

\section{Limitations}

Our study has limitations. While the MSB stain is more accurate than the H\&E stain in identifying major clot components, it still does not specifically identify other potentially key clot components such as vWF and calcification. Therefore, the authors represent this subgroup as 'platelet and other' components as we acknowledge that there are potentially other components in addition to platelets in these regions. Immunohistochemical analysis using specific antibodies is the only way to distinguish 
accurately between platelets and platelet-related factors such as $v$ WF. Also, the determination of stroke pathogenesis and etiology was self-reported at each site, and therefore there may have been some site-to-site variability in the interpretation and implementation of the TOAST criteria. For example, different sites likely had different thresholds to perform transesophageal versus transthoracic echocardiography or ultrasound bubble tests for CE sources. Lastly, there were no data on clinical outcome (ie, modified Rankin Scale) of patients included in the registry.

\section{CONCLUSIONS}

Our study of over 1300 retrieved emboli in ischemic stroke patients shows no consistent or reliable means to differentiate CE and LAA origin clots as determined by the MSB stain. Further research in this field should focus on more advanced techniques including immunohistochemistry and proteomic research.

\section{Author affiliations}

${ }^{1}$ Radiology, Mayo Clinic, Rochester, Minnesota, USA

${ }^{2}$ Neurosurgery, Mayo Clinic Rochester, Rochester, Minnesota, USA

${ }^{3}$ Neurology, Emory University School of Medicine, Atlanta, Georgia, USA

${ }^{4}$ Radiology, University of Tennessee Medical Center, Knoxville, Tennessee, USA

${ }^{5}$ Neurolnterventional Radiology, Baylor University Medical Center, Dallas, Texas, USA

${ }^{6}$ Interventional Neuroradiology, Abbot Northwestern Hospital, 55435, Minnesota,

USA

${ }^{7}$ Neurosurgery, Baptist Medical Center Jacksonville, Jacksonville, Florida, USA

${ }^{8}$ Division of Neuroradiology, Department of Medical Imaging and Division of Neurosurgery, Department of Surgery, University Health Network - Toronto Western Hospital, Toronto, Ontario, Canada

${ }^{9}$ Clinical Neurosciences, University of Calgary, Calgary, Alberta, Canada

${ }^{10}$ Neurointervention, Texas Stroke Institute, Plano, Texas, USA

${ }^{11}$ Neurosurgery and Radiology, Northwestern University, Chicago, Illinois, USA

${ }^{12}$ Radiology, New England Center for Stroke Research, University of Massachusetts

Medical School, Worcester, Massachusetts, USA

${ }^{13}$ Radiology, Neurosurgery, Carilion Clinic, Roanoke, Virginia, USA

${ }^{14}$ CÚRAM-SFI Research Centre for Medical Devices, National University of Ireland Galway, Galway, Ireland

${ }^{15}$ Physiology Department, National University of Ireland Galway, Galway, Ireland

${ }^{16}$ Radiology, University of Massachusetts, Worcester, Massachusetts, USA

${ }^{17}$ Division of Biomedical Statistics and Informatics, Mayo Clinic College of Medicine, Rochester, MN, USA

Twitter Vitor Mendes Pereira @VitorMendesPer1, Mohammed A Almekhlafi @ AlmekhlafiMa, Seán Fitzgerald @FitzSeanT and Pouya Nazari @PouyaNazari5

Contributors All authors have reviewed and approved the contents of this manuscript. This study is the result of a multicenter registry requiring the collaboration of multiple neurointerventionalists, research fellows and histopathologists and all authors meet the ICJME criteria for authorship.

Funding This work was supported by the National Institutes of Health grant number (R01 NS105853).

Competing interests None declared.

Patient consent for publication Obtained.

Provenance and peer review Not commissioned; externally peer reviewed.

Data availability statement Data pertaining to this study are available upon reasonable request to the corresponding author.

ORCID iDs

Waleed Brinjikji http://orcid.org/0000-0001-5271-5524
Ricardo A Hanel http://orcid.org/0000-0001-7195-5806

Vitor Mendes Pereira http://orcid.org/0000-0002-6804-3985

Mohammed A Almekhlafi http://orcid.org/0000-0001-9550-8197

Babak S Jahromi http://orcid.org/0000-0001-5064-5278

Matthew J Gounis http://orcid.org/0000-0002-8034-2785

Mehdi Abbasi http://orcid.org/0000-0001-6978-2563

Seán Fitzgerald http://orcid.org/0000-0001-6634-092X

Oana Madalina Mereuta http://orcid.org/0000-0003-3741-3784

Daying Dai http://orcid.org/0000-0003-4051-6450

Ramanathan Kadirvel http://orcid.org/0000-0002-6786-9953

Karen Doyle http://orcid.org/0000-0001-8984-6296

Alhamza R Al-Bayati http://orcid.org/0000-0001-8103-1930

Mahmoud H Mohammaden http://orcid.org/0000-0002-7393-9989

Gabriel Martins Rodrigues http://orcid.org/0000-0002-9152-0882

Pouya Nazari http://orcid.org/0000-0003-1135-1095

\section{REFERENCES}

1 Brinjikji W, Duffy S, Burrows A, et al. Correlation of imaging and histopathology of thrombi in acute ischemic stroke with etiology and outcome: a systematic review. J Neurointerv Surg 2017;9:529-34.

2 Brouwer PA, Brinjikji W, De Meyer SF. Clot pathophysiology: why is it clinically important? Neuroimaging Clin N Am 2018;28:611-23.

3 De Meyer SF, Andersson T, Baxter B, et al. Analyses of thrombi in acute ischemic stroke: a consensus statement on current knowledge and future directions. Int J Stroke 2017;12:606-14.

4 Fitzgerald S, Mereuta OM, Doyle KM, et al. Correlation of imaging and histopathology of thrombi in acute ischemic stroke with etiology and outcome. J Neurosurg Sci 2019;63:292-300.

5 Benson JC, Fitzgerald ST, Kadirvel R, et al. Clot permeability and histopathology: is a clot's perviousness on CT imaging correlated with its histologic composition? J Neurointerv Surg 2020;12:38-42.

6 Fitzgerald S, Rossi R, Mereuta OM, et al. Per-pass analysis of acute ischemic stroke clots: impact of stroke etiology on extracted clot area and histological composition. J Neurointerv Surg 2020;51:neurintsurg-2020-016966.

7 Mereuta OM, Fitzgerald S, Christensen TA, et al. High-resolution scanning electron microscopy for the analysis of three-dimensional ultrastructure of clots in acute ischemic stroke. J Neurointerv Surg 2020;63:neurintsurg-2020-016709.

8 Berndt M, Friedrich B, Maegerlein C, et al. Thrombus permeability in admission computed tomographic imaging indicates stroke pathogenesis based on thrombus histology. Stroke 2018;49:2674-82.

9 Boeckh-Behrens T, Kleine JF, Zimmer C, et al. Thrombus histology suggests cardioembolic cause in cryptogenic stroke. Stroke 2016;47:1864-71.

10 Fitzgerald S, Dai D, Wang S, et al. Platelet-rich emboli in cerebral large vessel occlusion are associated with a large artery atherosclerosis source. Stroke 2019;50:1907-10.

11 Sporns PB, Hanning U, Schwindt W, et al. Ischemic stroke: what does the histological composition tell us about the origin of the thrombus? Stroke 2017;48:2206-10.

12 Fitzgerald S, Wang S, Dai D, et al. Orbit image analysis machine learning software can be used for the histological quantification of acute ischemic stroke blood clots. PLOS One 2019;14:e0225841.

13 van der Laan MJ, Polley EC, Hubbard AE. Super learner. Stat App/ Genet Mol Biol 2007;6.

14 LeDell E, Gill N, Aiello S, et al. h2O: R Interface for the 'H2O 'Scalable Machine Learning Platform. R package version 2020;3.

15 Kim SK, Yoon W, Kim TS, et al. Histologic analysis of retrieved clots in acute ischemic stroke: correlation with stroke etiology and gradient-echo MRI. AJNR Am J Neuroradiol 2015;36:1756-62.

16 Muñoz R, Santamaría E, Rubio I, et al. Mass spectrometry-based proteomic profiling of thrombotic material obtained by endovascular thrombectomy in patients with ischemic stroke. Int J Mol Sci 2018;19:498.

17 Prochazka V, Jonszta T, Czerny D, et al. The role of von Willebrand factor, ADAMTS13, and cerebral artery thrombus composition in patient outcome following mechanical thrombectomy for acute ischemic stroke. Med Sci Monit 2018;24:3929-45.

18 Di Meglio L, Desilles J-P, Ollivier V, et al. Acute ischemic stroke thrombi have an outer shell that impairs fibrinolysis. Neurology 2019;93:e1686-98. 